\title{
Mortality in bronchiectasis: a long-term study assessing the factors influencing
} survival

\author{
M.R. Loebinger*, A.U. Wells", D.M. Hansell", N. Chinyanganya*, A. Devaraj", \\ M. Meister ${ }^{\top}$ and R. Wilson*
}

ABSTRACT: There is little literature about the mortality associated with bronchiectasis. The aim of the present study was to investigate factors affecting mortality in patients with bronchiectasis.

In total, 91 patients were examined for aetiology, pulmonary function tests, high-resolution computed tomography, sputum microbiology and quality of life scores and were then followed over 13 yrs.

Overall, $29.7 \%$ of the patients died. On multivariate analysis, age, St George's Respiratory Questionnaire activity score, Pseudomonas aeruginosa infection, total lung capacity (TLC), residual volume/TLC and the transfer factor coefficient were all independently associated with mortality.

In patients with moderate to severe bronchiectasis, mortality is associated with a degree of restrictive and obstructive disease, poor gas transfer and chronic pseudomonas infection. These features should guide future research into disease progression, and identify those patients needing intensive treatment.

KEYWORDS: Bronchiectasis, mortality, prognosis

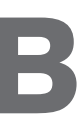
ronchiectasis is a chronic respiratory condition characterised by the abnormal dilatation of the bronchial lumen [1]. Damaged airways predispose the patient to recurrent bacterial infections, which in more severe cases become chronic. This leads to a host inflammatory response that causes tissue damage, leading to a vicious circle of host-mediated, bacteria-stimulated disease progression [2]. However, why in the majority of patients such progression is slow, whereas in others it occurs much more quickly is poorly understood.

Investigation of bronchiectasis aims to identify possible aetiologies and predisposing factors that may be treatable [3]. Management then centres on physiotherapy and antibiotics are used promptly in infective exacerbations. There is a lack of good randomised controlled trials for evidence-based management in bronchiectasis, and many of the management programmes are extrapolated from the treatment of other conditions such as pneumonia and cystic fibrosis [4].

A clearer picture of the natural history and mortality in bronchiectasis would be of great value, both in allowing more accurate prognostic information and in determining the specific characteristics of the disease that confer an increased risk of progression and mortality. This information will form the basis of future studies assessing the impact of therapies in bronchiectasis. Neutrophilic inflammation is thought to be a key component of the pathogenesis of chronic obstructive pulmonary disease (COPD), a condition in which infective exacerbations have been linked to disease progression $[5,6]$ and in which bronchiectasis is quite commonly seen in severe cases on computed tomography (CT) scans [7]. A better understanding of disease mechanisms in bronchiectasis might also improve COPD research.

In 1994, 111 patients with bronchiectasis were studied to validate the St George's Respiratory Questionnaire (SGRQ) as a tool in assessing the health status of patients with bronchiectasis [8] In the current study, 14 yrs on, we have reassessed these patients and carried out an analysis of the factors in the original assessment that predict mortality. The purpose of the present study was to provide prognostic information at baseline.

\section{METHODS}

In the original study in 1994, 120 sequential patients with clinically diagnosed bronchiectasis
AFFILIATIONS

*Host Defence Unit,

\# Interstitial Lung Disease Dept, and - Radiology Dept, Royal Brompton Hospital, London, UK.

CORRESPONDENCE

R. Wilson

Host Defence Unit

Royal Brompton Hospital

Sydney Street

London

SW3 6NP

UK

E-mail: r.wilson@rbht.nhs.uk

Received:

Jan 082009

Accepted after revision:

March 312009

First published online:

April 082009 
were approached to take part in this outpatient study in the Host Defence Unit of the Royal Brompton Hospital (London, UK), a tertiary referral centre. Of these, 111 agreed to take part in the study. The exclusion factors were bronchiectasis not being the predominant pathology and cystic fibrosis; however, no patients were in these groups [8]. Each patient provided signed consent and the protocols were approved by the Royal Brompton Hospital Ethics Committee. Each patient had full host defence investigations, which have been described elsewhere [9], including sweat testing for cystic fibrosis, two sputum samples that were also tested for nontuberculous mycobacteria, and a high-resolution CT scan. Comprehensive lung function tests with body plethysmography were performed on two separate occasions 6 months apart at entry to the study.

The outcome of these patients was determined as of March 2007, 13 yrs after the initial recruitment. Patients not followed up in our institution were tracked via general practitioners, other hospitals and the Office for National Statistics, and death certificates were obtained for all patients that died. The CT scans were reassessed and on this basis the patient number was reduced further to only include patients who also had bronchiectasis according to CT criteria.

CT images of $1-1.5 \mathrm{~mm}$ thickness at $10 \mathrm{~mm}$ intervals were scored for bronchiectasis $[10,11]$ by two blinded, independent radiologists. The scoring system used was modified from the system of BHALLA et al. [12] and has been shown to be associated with low inter-observer variation [13]. Bronchiectasis extent and bronchial wall thickness were scored in each lobe on a scale of 0 to 3 (3: most severe changes). Bronchial dilatation and mucus plugging were graded from 0 to 2 . A mosaic attenuation pattern (reflecting small airways obliteration) and emphysema were scored to the nearest $5 \%$. The average score of the two observers, as a percentage of the maximum score possible to account for patients with lobectomies, was used for analysis.

\section{Statistical analysis}

Kaplan-Meier curves were used to illustrate survival data. Variables were examined against survival using Cox's proportional hazards model: in multivariate models, a stepwise approach was used to discard variables not independently linked to mortality $(p>0.05)$. Hazard ratios are given with confidence intervals (CIs) for significant $(\mathrm{p}<0.05)$ and marginal $(0.05<\mathrm{p}<0.15)$ trends.

Stepwise multiple linear regression was used to identify independent CT determinants of lung function impairment. Lung function indices shown to have an independent impact on mortality (total lung capacity (TLC), residual volume (RV)/ TLC and transfer coefficient of the lung for carbon monoxide $(\mathrm{KCO})$ ) were used as the dependent variables in separate models. The validity of the parametric assumptions of linear regression was confirmed with testing for heteroscedasticity and RV/TLC was subjected to zero skewness logarithmic transformation on the basis of this test.

Statistical analyses were performed using STATA (version 4; StataCorp LP, College Station, TX, USA).

\section{RESULTS}

\section{Patient demographics and survival}

There were 111 patients that consented to the original SGRQ study in 1994. Review of CTs found no bronchiectasis in 12 patients and these were not included in further analysis. These patients had a clinical presentation compatible with bronchiectasis, but they did not satisfy the CT criteria. The data set was incomplete in a further eight patients and they were also excluded, leaving 91 patients in the study (92\% of the eligible patients; fig. 1). Patient demographics are shown in table 1.

Patients were followed up from recruitment in 1994, until March 2007. During the 13-yr follow-up, $29.7 \%$ of the bronchiectasis patients died. Using life expectancy data from the Office for National Statistics, the expected death rate of a 52 -yr-old (mean age in our study) is $14.7 \%$ for males and $8.9 \%$ females over a similar 13-yr period [14]. Accounting for the mortality in our study, the mean \pm SD length of follow-up was $126.9 \pm 38.0$ months. All the 91 patients were successfully accounted for at the end of the study. The cause of death was bronchiectasis, respiratory infection or respiratory failure in $19(70.4 \%)$ out of 27 cases. Haemoptysis was the terminal event in one of these patients. Two patients each died of renal failure and colon cancer, and one patient each died of heart failure, cerebrovascular accident, liver metastasis and pulmonary embolism. Post mortem examinations were performed in three patients and the cause of death was classified as suppurative bronchitis, bronchiectasis and heart failure. Of those who died during the study, the median age was 60 yrs. A Kaplan-Meier survival plot is shown in figure 2.

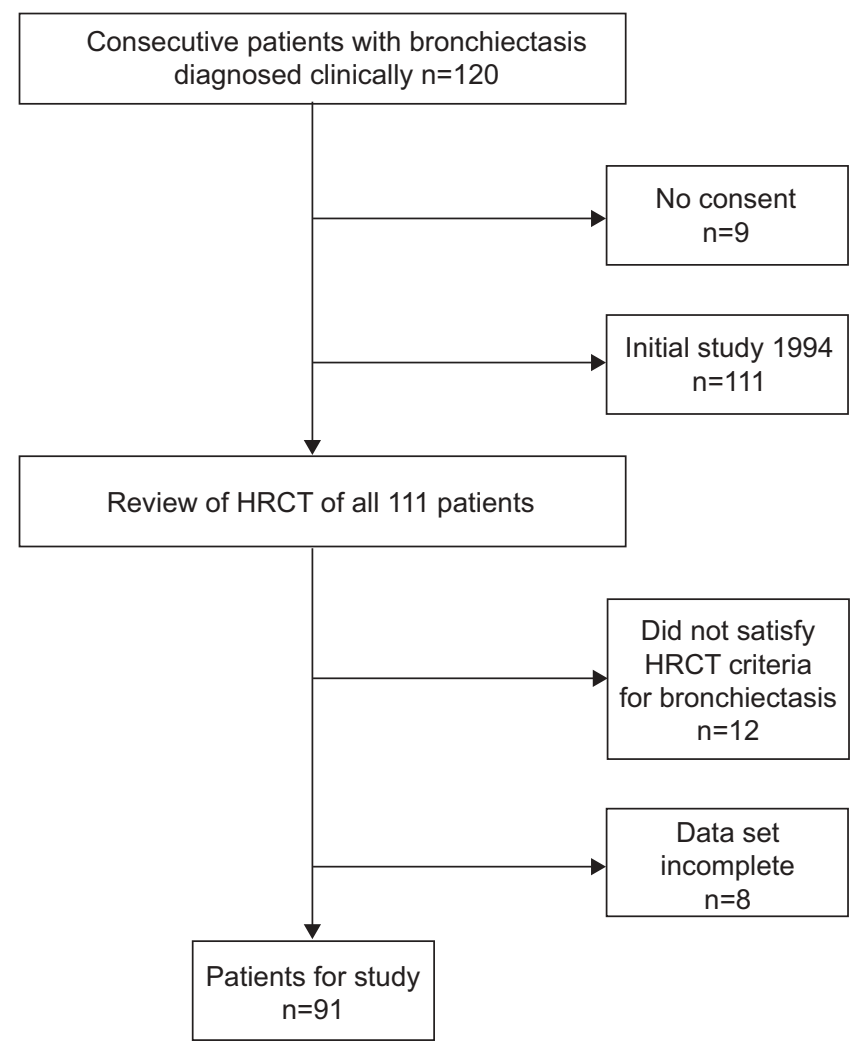

FIGURE 1. Flow diagram depicting patient selection. HRCT: high-resolution computed tomography. 


\begin{tabular}{|c|c|c|}
\hline TABLE 1 & \multicolumn{2}{|c|}{$\begin{array}{l}\text { General characteristics of study patients with } \\
\text { bronchiectasis }\end{array}$} \\
\hline \multicolumn{2}{|l|}{ Subjects n } & 91 \\
\hline \multicolumn{2}{|c|}{ Mortality dead/alive } & $27(29.7) / 64(70.3)$ \\
\hline \multicolumn{2}{|c|}{ Sex female/male } & $53(58.2) / 38(41.8)$ \\
\hline \multicolumn{2}{|c|}{ Smoker current/former/never } & $1(1.1) / 20(22) / 70(76.9)$ \\
\hline \multicolumn{2}{|c|}{ PSA/non-PSA } & $20(22) / 71(78)$ \\
\hline \multicolumn{2}{|c|}{ Age in 1994 yrs } & $51.7 \pm 12.1$ \\
\hline \multicolumn{2}{|c|}{ Length of follow-up ${ }^{\#}$ months } & $126.9 \pm 38.0$ \\
\hline \multicolumn{2}{|c|}{ Period between CT and LF months } & $13.0 \pm 14.1$ \\
\hline \multicolumn{3}{|c|}{ CT criteria ${ }^{\circ}$} \\
\hline \multicolumn{2}{|c|}{ Bx extent } & $33.1 \pm 21.0$ \\
\hline \multicolumn{2}{|c|}{ Dilatation severity } & $39.7 \pm 24.8$ \\
\hline \multicolumn{2}{|c|}{ Wall thickness } & $20.6 \pm 12.4$ \\
\hline \multicolumn{2}{|c|}{ Small airway plugging } & $9.8 \pm 14.7$ \\
\hline \multicolumn{2}{|c|}{ Large airway plugging } & $12.5 \pm 13.1$ \\
\hline \multicolumn{2}{|c|}{ Mosaicism } & $7.5 \pm 10.2$ \\
\hline \multicolumn{2}{|c|}{ Emphysema } & $3.9 \pm 8.0$ \\
\hline \multicolumn{3}{|c|}{ LF criteria \% pred } \\
\hline \multicolumn{2}{|c|}{ FEV 1} & $65.8 \pm 28.1$ \\
\hline \multicolumn{2}{|l|}{ FVC } & $88.7 \pm 23.6$ \\
\hline \multicolumn{2}{|l|}{$\mathrm{FEV}_{1} / \mathrm{FVC}$} & $83.1 \pm 9.0$ \\
\hline \multicolumn{2}{|l|}{ RV } & $136.8 \pm 44.1$ \\
\hline \multicolumn{2}{|l|}{ TLC } & $103.1 \pm 16.6$ \\
\hline \multicolumn{2}{|l|}{$\mathrm{RV} / \mathrm{TLC}$} & $126.9 \pm 31.3$ \\
\hline \multicolumn{2}{|l|}{$T L, \mathrm{CO}$} & $76.2 \pm 20.4$ \\
\hline \multicolumn{2}{|l|}{ KCO } & $95.4 \pm 22.1$ \\
\hline \multicolumn{3}{|c|}{$\begin{array}{l}\text { Health-related quality of life } \\
\text { questionnaire scores }\end{array}$} \\
\hline Fatigue & & $4.7 \pm 2.4$ \\
\hline MRC whee & & $2.2 \pm 1.3$ \\
\hline MRC dyspr & $\mathrm{ea}^{+}$ & $2.2 \pm 1.0$ \\
\hline SGRQ total & & $45.9 \pm 18.1$ \\
\hline SGRQ sym & toms $s^{\S}$ & $71.9 \pm 18.8$ \\
\hline SGRQ acti & $i^{i} s^{\S}$ & $49.1 \pm 24.5$ \\
\hline SGRQ imp & & $35.7 \pm 19.0$ \\
\hline Aetiology & & \\
\hline Idiopathic & & $51(56)$ \\
\hline Post infecti & & $20(22)$ \\
\hline ABPA & & $8(8.8)$ \\
\hline PCD & & $5(5.5)$ \\
\hline Young's sy & drome & $4(4.4)$ \\
\hline Hypogamm & globulinaemia & $3(3.3)$ \\
\hline
\end{tabular}

Data are presented as $n(\%)$ or mean $\pm \mathrm{SD}$, unless otherwise stated. Data are presented from time of entry into the cohort. PSA: Pseudomonas aeruginosa; CT: computed tomography; LF: lung function; Bx: bronchiectasis; \% pred: \% predicted; FEV1: forced expiratory volume in $1 \mathrm{~s}$; FVC: forced vital capacity; RV: residual volume; TLC: total lung capacity; $T L, C O$ : transfer factor of the lung for carbon monoxide; $\mathrm{KCO}$ : transfer coefficient of the lung for carbon monoxide; MRC: Medical Research Council; SGRQ: St George's Respiratory Questionnaire; ABPA: allergic bronchopulmonary aspergillosis; PCD: primary ciliary dyskinesia. \#: from recruitment until March 1, 2007, or until patient died; $\because$ : expressed as a percentage of the maximal possible score for each criterion; ${ }^{+}$: range $1-5$, with a score of 1 representing greatest breathlessness on strenuous exercise; ${ }^{\text {s. }}$ : range 0-100, with a zero score indicating no impairment in quality of life.

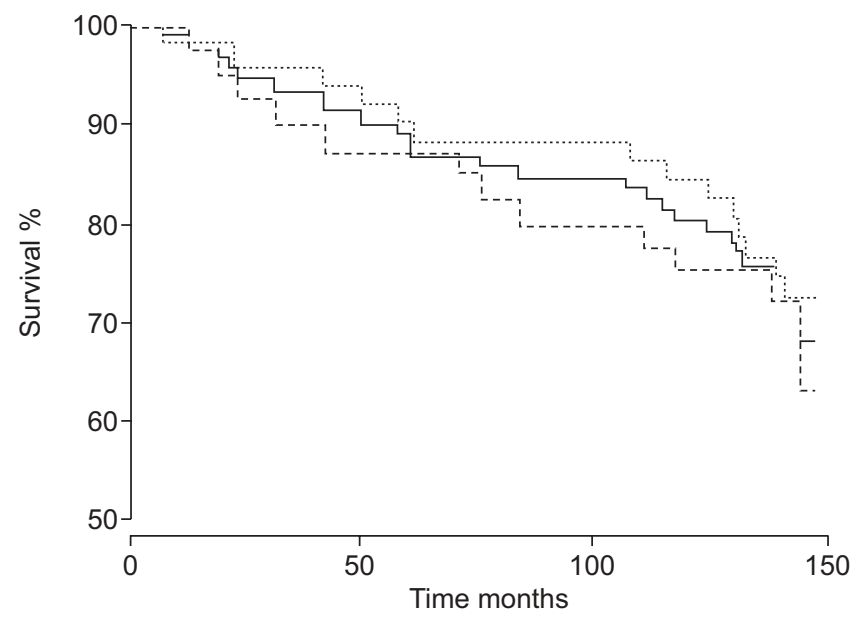

FIGURE 2. Kaplan-Meier plot illustrating the survival of all the bronchiectasis patients $(-)$, in addition to the idiopathic $(\cdots \cdots)$ and known $(----)$ aetiology subgroups. There are no statistically significant differences between the plots (log rank test; $p=0.85$ )

The aetiology of the bronchiectasis was as defined at recruitment and was similar to previous studies [9, 15]. Idiopathic aetiology accounted for more cases $(56.0 \%)$ than a recent study (34.6\%) [9]. Nevertheless, the same broad trends were observed, with idiopathic and post-infective causes of bronchiectasis being the most common aetiologies. The majority of the patients in the current study were neversmokers $(76.9 \%)$ and the proportion of emphysema on the high-resolution CT scans was also low (mean score $<4 \%$ ). There was a range of severity scores (table 1), suggesting that the present study population covered a spectrum of disease activity; however, broadly the cohort described a population with moderate to severe bronchiectasis. Bronchiectasis affected a total of $304(56.8 \%)$ out of a possible 535 lobes examined on CT scan. Spirometry showed that airflow obstruction was the predominant finding in this group of bronchiectasis patients, with an average forced expiratory volume in $1 \mathrm{~s}$ (FEV1) of $1.85 \mathrm{~L} \cdot \mathrm{min}^{-1}(65.8 \%$ predicted $)$ and forced vital capacity of $3.05 \mathrm{~L}(88.7 \%$ pred), which is in accordance with other studies [15-17].

\section{Mortality risk factors}

All the measured variables were included in univariate analysis, as shown in table 2 . A relative rate (RR) $>1$ describes a positive correlation between the parameter and mortality, whereas $R R<1$ suggests a negative correlation. There were large ranges of lung function, CT and health status variables that had a strong relationship to mortality in the current analysis. There were particularly strong associations for bronchiectasis extent, bronchiectasis severity and wall thickness on CT assessment; FEV1 and transfer factor of the lung for carbon monoxide on lung function measurement; and dyspnoea and activity score on the health status assessment. The RRs and CIs for the CT and lung function criteria appear much lower than, for example, the existence of pseudomonas. This is a function of them being continuous as opposed to categorical variables. For lung function tests, a risk ratio reflects a change in risk per unit lung function. In other words, a risk ratio of 0.97 for FEV1 means that for every $1 \%$ change in FEV1 there is a 


\begin{tabular}{|c|c|c|}
\hline Parameters & RR $(95 \% \mathrm{Cl})^{\#}$ & $p$-value \\
\hline Male sex & $1.96(0.92-4.20)$ & 0.082 \\
\hline Age & $1.06(1.02-1.09)$ & 0.001 \\
\hline Smoker & $1.78(0.87-3.63)$ & 0.120 \\
\hline PSA & $2.33(1.04-5.18)$ & 0.039 \\
\hline \multicolumn{3}{|l|}{ CT criteria } \\
\hline Bx extent & $1.04(1.02-1.05)$ & $<0.0005$ \\
\hline Dilatation severity & $1.03(1.01-1.04)$ & $<0.0005$ \\
\hline Wall thickness & $1.07(1.04-1.10)$ & $<0.0005$ \\
\hline Small airway plugging & & 0.506 \\
\hline Large airway plugging & $1.04(1.02-1.07)$ & 0.002 \\
\hline Mosaicism & $1.03(1.00-1.06)$ & 0.049 \\
\hline Emphysema & $1.05(1.02-1.08)$ & 0.001 \\
\hline \multicolumn{3}{|l|}{ LF criteria \% pred } \\
\hline FEV 1 & $0.97(0.95-0.98)$ & $<0.0005$ \\
\hline FVC & $0.98(0.96-0.99)$ & 0.003 \\
\hline $\mathrm{FEV}_{1 / \mathrm{FVC}}$ & & 0.209 \\
\hline RV & & 0.176 \\
\hline TLC & $0.98(0.96-1.01)$ & 0.192 \\
\hline $\mathrm{RV} / \mathrm{TLC}$ & $1.01(1.00-1.02)$ & 0.021 \\
\hline$T L, C O$ & $0.95(0.93-0.97)$ & $<0.0005$ \\
\hline KCO & $0.98(0.97-0.99)$ & 0.007 \\
\hline \multicolumn{3}{|c|}{$\begin{array}{l}\text { Health-related quality of life } \\
\text { questionnaire scores }\end{array}$} \\
\hline Fatigue & $1.14(0.97-1.35)$ & 0.122 \\
\hline MRC wheeze & $1.26(0.92-1.71)$ & 0.147 \\
\hline MRC dyspnoea & $2.89(1.84-4.54)$ & $<0.0005$ \\
\hline SGRQ total & $1.04(1.01-1.06)$ & 0.002 \\
\hline SGRQ symptoms & $1.02(1.00-1.04)$ & 0.121 \\
\hline SGRQ activities & $1.04(1.02-1.06)$ & $<0.0005$ \\
\hline SGRQ impacts & $1.02(1.00-1.04)$ & 0.035 \\
\hline
\end{tabular}

RR: relative rate; Cl: confidence interval; PSA: Pseudomonas aeruginosa; CT: computed tomography; Bx: bronchiectasis; LF: lung function; \% pred: \% predicted; FEV1: forced expiratory volume in $1 \mathrm{~s}$; FVC: forced vital capacity; RV: residual volume; TLC: total lung capacity; $T L, C O$ : transfer factor of the lung for carbon monoxide; $\mathrm{KCO}$ : transfer coefficient of the lung for carbon monoxide; MRC: Medical Research Council; SGRQ: St George's Respiratory Questionnaire. ${ }^{\#}$ : RR $>1$ describes a positive correlation between the parameter and mortality, whereas $\mathrm{RR}<1$ suggests a negative correlation. Entries in bold are statistically significant.

$3 \%$ change in risk. A difference of $30 \%$ FEV1 consequently confers a relative rate of 2.71 (95\% CI 1.65-4.45).

Several of these variables will represent different ways of measuring the same effect. The multivariate analysis demonstrated the variables with independent effects on mortality. Complex CT scoring systems are not practical for routine clinical practice and would not be available in most centres, and thus these variables were excluded from the multivariate analysis. Increased age, male sex, Pseudomonas aeruginosa colonisation and higher SGRQ activity scores (describing lower activity levels), in addition to higher RV/TLC and lower TLC, and KCO lung function measurements were independent predictors of mortality (table 3 ).

\begin{tabular}{llc}
\hline TABLE 3 & $\begin{array}{l}\text { Multivariate Cox proportional hazard stepwise } \\
\text { analysis }\end{array}$ & p-value \\
\hline Parameters & RR (95\% Cl) & \\
\hline Age & $1.10(1.06-1.15)$ & $<0.0005$ \\
PSA & $3.61(1.35-9.62)$ & 0.010 \\
Male sex & $3.42(1.34-8.77)$ & 0.010 \\
LF criteria \% pred & $1.03(1.01-1.04)$ & $<0.0005$ \\
$\quad$ RV/TLC & $0.95(0.93-0.98)$ & $<0.0005$ \\
$\quad$ TLC & $0.96(0.94-0.98)$ & $<0.0005$ \\
$\quad$ KCO & & \\
Health-related quality of life & & $<0.0005$ \\
$\quad$ questionnaire scores & $1.05(1.02-1.08)$ & \\
$\quad$ SGRQ activities & & \\
\hline
\end{tabular}

RR: relative rate; Cl: confidence interval; PSA: Pseudomonas aeruginosa; LF: lung function; \% pred: \% predicted; RV: residual volume; TLC: total lung capacity; KCO: transfer coefficient for the lung for carbon monoxide; SGRQ: St George's Respiratory Questionnaire. \#: RR $>1$ describes a positive correlation between the parameter and mortality, whereas $R R<1$ suggests a negative correlation.

Although not used in the main multivariate model, the CT variables were assessed together in a separate multivariate analysis to find the most important CT factors that had strong independent effects on mortality. Increased wall thickness (RR $1.06,95 \%$ CI 1.03-1.10; $\mathrm{p}<0.005$ ) and emphysema (RR 1.04, 95\% CI 1.00-1.07; $p=0.027)$, despite the low prevalence of this latter feature, were the strongest predictors of mortality in bronchiectasis from the CT findings.

Seven patients had lobectomies and four of these died. The mean \pm SD TLC in the lobectomy group was $4.63 \pm 0.91 \mathrm{~L}$, which was $80.43 \pm 10.97 \%$ pred, compared with $6.00 \pm 1.42 \mathrm{~L}$ $(105.04 \pm 15.55 \%$ pred) in the other patients. However, the lung function parameters that predicted mortality in the survival analysis were unchanged when these seven patients were removed.

There was no difference in mortality rates among the different aetiologies of bronchiectasis. As the number in several of the groups was very small, we separated them into idiopathic and known causes $(p=0.57$ Cox proportional hazard) and have illustrated mortality differences using Kaplan-Meier curves (fig. 2)

\section{Functional-morphological correlations}

The lung function measures that independently predict survival in bronchiectasis are TLC, RV/TLC and KCO, as shown previously. The morphological correlates of these were examined by separate linear regression models with these three lung function measures as the dependent variables and the CT variables as cofactors. A regression coefficient $(\mathrm{RC})>0$ describes a positive correlation between the parameter measurements, whereas $\mathrm{RC}<0$ suggests a negative correlation. TLC was positively correlated to mosaicism on the CT scan, RV/TLC was positively correlated with wall thickness and mosaicism, and KCO was negatively correlated with emphysema (table 4). 


\begin{tabular}{|c|c|c|}
\hline TABLE 4 & \multicolumn{2}{|c|}{$\begin{array}{l}\text { Independent relationships between computed } \\
\text { tomography measurements and the lung } \\
\text { function measurements shown to correlate with } \\
\text { mortality }\end{array}$} \\
\hline Parameters & $\mathrm{RC}(95 \% \mathrm{Cl})^{\#}$ & $p$-value \\
\hline \multicolumn{3}{|l|}{ TLC \% pred } \\
\hline Mosaicism & $0.41(0.06-0.75)$ & 0.02 \\
\hline \multicolumn{3}{|c|}{ RV/TLC \% pred log" } \\
\hline Wall thicknes & $0.02(0.01-0.02)$ & $<0.0005$ \\
\hline Mosaicism & $0.01(0.01-0.02)$ & 0.001 \\
\hline \multicolumn{3}{|l|}{ Kco \% pred } \\
\hline Emphysema & $-0.85(-1.4--0.30)$ & 0.003 \\
\hline \multicolumn{3}{|c|}{$\begin{array}{l}\text { RC: regression coefficient; Cl: confidence interval; TLC: total lung capacity; } \\
\% \text { pred: \% predicted; RV: residual volume; Kco: transfer coefficient of the lung } \\
\text { for carbon monoxide. }{ }^{\#}: \mathrm{RC}>0 \text { describes a positive correlation between the } \\
\text { parameter measurements, whereas } \mathrm{RC}<0 \text { suggests a negative correlation; } \\
\text { : log transformed (log } 56.93 \text { ) to correct for skewness. }\end{array}$} \\
\hline
\end{tabular}

\section{Longitudinal data}

The purpose of the current study was to construct prognostic indices from baseline data. We did not routinely repeat these tests during the course of the study. Nevertheless, the majority of patients did have several repeat lung function and sputum investigations during follow-up. There was an average decrease in KCO per year of follow-up of 0.03 (95\% CI $0.01-$ $0.04)$. This was significantly greater in those who died compared with the surviving patients $(0.05(0.005-0.09)$ versus 0.02 (0.004-0.03), respectively; $p=0.02$, Mann-Whitney). The longitudinal changes in TLC $\left(0.02(-0.02-0.06) \mathrm{yr}^{-1}\right)$ and RV/ TLC $\left(0.1 \%(-0.62-0.82 \%) \mathrm{yr}^{-1}\right)$ were insignificant, with no difference between the patients that survived or died. Repeat sputum cultures were also performed in 17 out of the 20 patients with $P$. aeruginosa. Of these, $P$. aeruginosa was a consistent finding in 11 out of 17 patients with intermittent cultures in six out of 17 patients.

\section{DISCUSSION}

Prior to the present study, the outcome in adult bronchiectasis had not been well described and there has been a lack of longterm prospective studies. The current study is unique in having a very well defined bronchiectatic population, all of whom had extensive measurements of disease at baseline. Furthermore, all patients were followed prospectively until death or the end of the study 13 yrs later. The extensive nature of the testing in the present study and the length of follow-up mean that it is ideally placed to comment on features of bronchiectasis patients that influence mortality.

The average age of death in patients with bronchiectasis was found to be $<55$ yrs in a study in the 1960s [18], and most patients succumbed to the disease before the age of $40 \mathrm{yrs}$ in a report of 400 bronchiectatics in 1940 [19]. Since this time, however, the condition, aetiologies and treatment have altered with the advent of vaccination programmes and the increased use of antimicrobials for childhood infections and tuberculosis. More recent studies have reported a variation in mortality rates in bronchiectasis, with the worst being a 4-yr survival of 58\% [16], a retrospective study showing a $75 \%$ survival at 8.8 yrs [20], and the best being an $81 \%$ survival at 14 yrs [17]. The survival rates during our study were broadly comparable to the latter two studies, being $91 \%$ at 4 yrs, $83.5 \%$ at 8.8 yrs and $68.3 \%$ at the end of 12.3 yrs (fig. 2). The primary cause of death in our patients was respiratory, suggesting that patients die from, not just with, bronchiectasis.

The current study looked specifically at the factors associated with mortality rate in bronchiectasis over a 13-yr period. There are few previous studies assessing this, but a poor initial ventilatory capacity [17], male sex [20], and a secondary diagnosis such as asthma or COPD [20] have all been suggested as inferring a worse prognosis in bronchiectasis. A more recent report prospectively followed 98 patients with bronchiectasis over a 4-yr period in Turkey. Risk factors for mortality in this study were only looked at in a univariate analysis, and included age, low body mass index, hypoxia, hypercapnia, radiographic extent, Medical Research Council dyspnoea scale and lack of vaccinations [16]. The results of our study have demonstrated that the factors with an independent impact on mortality are age, SGRQ activity score, $P$. aeruginosa colonisation, and TLC, $\mathrm{RV} / \mathrm{TLC}$ and KCO lung function measurements.

Among a larger number of variables examined against mortality, most were not independently predictive and were discarded using a stepwise methodology, to develop a final model that contained only six covariates. Failure to discard nonsignificant variables would have resulted in confounding by collinearity (as, for example, between pulmonary function measures of airflow obstruction), as well as a degree of model over-fitting. However, it should also be acknowledged that in multivariate analysis, the retention of one collinear variable (quantifying, for example, airflow obstruction), as opposed to another, is sometimes a very close call. The multivariate modelling strategy used is predominantly for enabling mechanistic conclusions as opposed to predicting individual patient outcomes. The results should be interpreted as identifying the broad determinants of mortality (which includes the severity of airflow obstruction), in the search for pathogenetic insights. The model is not advanced as a multivariate clinical index for immediate use. Nevertheless, the stronger univariate relationships we report are of immediate clinical relevance.

Formal CT scoring, as performed in our study, is unrealistic in routine clinical practice [21] and was not included in final multivariate modelling. This approach was further validated by the finding that $\mathrm{CT}$ features did not reach statistical significance for an independent effect on mortality when reentered into the modelling equations. The CT variables are nevertheless very useful in order to appreciate the pathogenetic correlates of the functional indices. The CT scan correlates of the lung function parameters with an independent impact on mortality, TLC, RV/TLC and KCO were mosaicism, wall thickness and mosaicism, and emphysema, respectively.

Of the variables shown to have an independent effect on mortality in bronchiectasis, $P$. aeruginosa has previously been shown to infect patients with more extensive disease and severe airflow obstruction [22]. Two studies have shown that infection leads to an increased progression of disease [23] with a more rapid decline in lung function parameters [24]. 
The cause and effect relationship is less clear, as this pathogen may instead be a marker of disease progression as opposed to the cause for progression [25]. The independent effect of infection with $P$. aeruginosa on mortality shown in the present study suggests that it is likely to impact on survival more than just being a marker of severity. Infection control measures, such as clinic segregation, to avoid patient exposure to the bacterium, should be investigated. Furthermore, treatment regimes designed to eradicate $P$. aeruginosa, when it occurs for the first time, have shown some benefit in delaying the decline of lung function in patients with cystic fibrosis [26, 27], and may similarly impact on the progression of bronchiectasis [4]. This should be investigated in randomised controlled studies.

Poor lung function measurements have previously been shown to be associated with mortality in bronchiectasis [17]. Our study has shown an increased mortality in patients with a high $\mathrm{RV} / \mathrm{TLC}$ ratio. This suggests the importance of obstruction in the presence of restriction in this condition. In the multivariate model, RV/TLC was more important than the other variables that measured obstruction, suggesting that obstruction had the greatest effect on mortality in the presence of some restriction. The importance of a component of restrictive lung disease in bronchiectasis is also confirmed by the correlation between mortality and a lower TLC in the present study. This relationship was still present when those patients who had lobectomies were removed. The final lung function measurement with an independent effect on mortality in this study is KCO. This demonstrates a vascular or emphysematous component that impacts on mortality in bronchiectasis. The low prevalence of emphysema in CT scans suggests the former as the more likely. There appear to be separate and independent effects of the three individual components of functional impairment on lung function testing, with obstruction, restriction and impairment of gas transfer all separately implicated in mortality. These components are likely to represent separate pathogenic components of bronchiectasis that may be important in helping to understand the impact of disease, in addition to providing specific targets for future treatments. To elucidate the potential morphological correlates of these lung function measures, they were correlated with CT findings.

The measure of obstruction, RV/TLC, was correlated to mosaicism and wall thickness on CT scans. The correlation of functional obstruction to wall thickness does not differentiate between secretions and ablative bronchiolitis as the cause; however, the relationship with mosaicism favours the latter as a morphological explanation of the obstructive functional element in bronchiectasis. This is in agreement with a previous study correlating CT findings with FEV1, which is another measure of airways obstruction [28]. The authors concluded that airways obstruction in bronchiectasis was primarily related to intrinsic disease of small and medium airways, and not to the large airway abnormalities, airway mucus plugging or emphysema. Small airways disease is prominent in histological studies of bronchiectasis, with obstructive and inflammatory bronchiolitis leading to the features of airways obstruction and decreased radiographic attenuation [29].

In contrast, CT features could not explain the restrictive element, with TLC showing only a positive correlation with mosaicism. Possible pathological correlates of lung restriction in bronchiectasis include parenchymal scarring secondary to previous infections, pleural disease, peribronchial fibrosis and atelectasis. Peribronchial fibrosis has been demonstrated in histological studies of bronchiectasis [30], and this together with atelectasis distal to the ablated airways may not be detectable on CT scanning [28].

The contribution of poor gas exchange to mortality may be secondary to emphysema or to a pulmonary vascular component. The KCO was positively correlated to emphysema and not mosaicism, suggesting emphysema as the morphological correlate of low transfer factor. However, CT measurements of the pulmonary vasculature are poor, and the possible importance of pulmonary hypertension in bronchiectasis is further highlighted by an earlier study in which cor pulmonale was present in $37 \%$ of patients who died from bronchiectasis [17]. From a management point of view, the importance of assessing pulmonary artery pressures in bronchiectasis patients with low transfer factors should be emphasised, as these patients may be amenable to some of the newer anti-hypertensive agents.

In addition to helping understand the pathogenesis of the condition and providing additional targets for treatment, knowledge of the lung function factors associated with mortality may provide useful correlates for mortality against which future treatments can be tested, and it may be used in defining subgroups of patients into prognostic categories. The severity of bronchiectasis is presently defined by the extent and grade of large airway dilatation. These features are not associated with prognosis and hence a paradigm involving lung function may be more appropriate. The current study was not designed to investigate the rate of change of parameters over time and hence routine repeat measurements were not performed. The longitudinal investigations we have described were taken at different times and for different indications. With these limitations, a decline in KCO was demonstrated over time, predominantly in the patients that died, and this may be helpful in providing a basis for assessing the stability of patients in the long term.

In summary, the present study has suggested the importance of lung function measurements in addition to age, $P$. aeruginosa infection and health questionnaire activity scores in the prognosis of bronchiectasis. It is important to note that the conclusions refer to a tertiary centre and are subject to the limitation of a predominantly retrospective analysis. Nevertheless, knowledge of the results from the current study should change investigation protocols and define patients that need more intensive management plans, such as antibiotics or anti-inflammatory agents. The results may also help to define useful subgroups for the assessment of treatment regimes in much needed randomised controlled trials, to help advance bronchiectasis treatment towards a stronger evidence base.

\section{STATEMENT OF INTEREST}

None declared.

\section{ACKNOWLEDGEMENTS}

The current authors would like to acknowledge P. Jones (St George's Hospital, London, UK) and C. Wilson (no affiliation; previously Royal Brompton Hospital, London) for their major contribution to the initial investigation of the cohort of patients, and their helpful comments on the present work. 


\section{REFERENCES}

1 Barker AF. Bronchiectasis. N Engl J Med 2002; 346: 1383-1393.

2 Cole PJ. A new look at the pathogenesis and management of persistent bronchial sepsis: a vicious circle hypothesis and its logical therapeutic connatations. In: Davies RJ, ed. Strategies for the Management of Chronic Bronchial Sepsis. Oxford, Medicine Publishing Foundation, 1984; pp. 1-20.

3 Wilson R. Bronchiectasis. In: Brewis RAL, ed. Respiratory Medicine. 3rd Edn. London, WB Saunders, 2003; pp. 1445-1464.

4 Loebinger MR, Wilson R. Pharmacotherapy for bronchiectasis. Expert Opin Pharmacother 2007; 8: 3183-3193.

5 Papi A, Bellettato CM, Braccioni F, et al. Infections and airway inflammation in chronic obstructive pulmonary disease severe exacerbations. Am J Respir Crit Care Med 2006; 173: 1114-1121.

6 Wedzicha JA, Seemungal TA. COPD exacerbations: defining their cause and prevention. Lancet 2007; 370: 786-796.

7 Patel IS, Vlahos I, Wilkinson TM, et al. Bronchiectasis, exacerbation indices, and inflammation in chronic obstructive pulmonary disease. Am J Respir Crit Care Med 2004; 15, 170: 400-407.

8 Wilson CB, Jones PW, O'Leary CJ, et al. Validation of the St. George's Respiratory Questionnaire in bronchiectasis. Am J Respir Crit Care Med 1997; 156: 536-541.

9 Shoemark A, Ozerovitch L, Wilson R. Aetiology in adult patients with bronchiectasis. Respir Med 2007; 101: 1163-1170.

10 Grenier P, Cordeau MP, Beigelman C. High-resolution computed tomography of the airways. J Thorac Imaging 1993; 8: 213-229.

11 McGuinness G, Naidich DP. Bronchiectasis: CT/clinical correlations. Semin Ultrasound CT MR 1995; 16: 395-419.

12 Bhalla $\mathrm{M}$, Turcios $\mathrm{N}$, Aponte $\mathrm{V}$, et al. Cystic fibrosis: scoring system with thin-section CT. Radiology 1991; 179: 783-788.

13 Reiff DB, Wells AU, Carr DH, et al. CT findings in bronchiectasis: limited value in distinguishing between idiopathic and specific types. AJR Am J Roentgenol 1995; 165: 261-267.

14 Office for National Statistics. English Life Tables No.15. London, The Stationery Office, 1997. Available at www.statistics.gov.uk/ downloads/theme_population/ELT15.pdf

15 Pasteur MC, Helliwell SM, Houghton SJ, et al. An investigation into causative factors in patients with bronchiectasis. Am J Respir Crit Care Med 2000; 162: 1277-1284.

16 Onen ZP, Gulbay BE, Sen E, et al. Analysis of the factors related to mortality in patients with bronchiectasis. Respir Med 2007; 101: 1390-1397.
17 Ellis DA, Thornley PE, Wightman AJ, et al. Present outlook in bronchiectasis: clinical and social study and review of factors influencing prognosis. Thorax 1981; 36: 659-664.

18 Konietzko NF, Carton RW, Leroy EP. Causes of death in patients with bronchiectasis. Am Rev Respir Dis 1969; 100: 852-858.

19 Perry KMA, King DS. Bronchiectasis: a study of prognosis based on a follow-up of 400 patients. Am Rev Tuberc 1940; 41: 531-548.

20 Keistinen T, Saynajakangas O, Tuuponen T, et al. Bronchiectasis: an orphan disease with a poorly-understood prognosis. Eur Respir J 1997; 10: 2784-2787.

21 Goh NS, Desai SR, Veeraraghavan S, et al. Interstitial lung disease in systemic sclerosis: a simple staging system. Am J Respir Crit Care Med 2008; 177: 1248-1254.

22 Miszkiel KA, Wells AU, Rubens MB, et al. Effects of airway infection by Pseudomonas aeruginosa: a computed tomographic study. Thorax 1997; 52: 260-264.

23 Wilson CB, Jones PW, O'Leary CJ, et al. Systemic markers of inflammation in stable bronchiectasis. Eur Respir J 1998; 12 820-824.

24 Evans SA, Turner SM, Bosch BJ, et al. Lung function in bronchiectasis: the influence of Pseudomonas aeruginosa. Eur Respir J 1996; 9: 1601-1604.

25 Davies G, Wells AU, Doffman S, et al. The effect of Pseudomonas aeruginosa on pulmonary function in patients with bronchiectasis. Eur Respir J 2006; 28: 974-979.

26 Frederiksen B, Koch C, Hoiby N. Antibiotic treatment of initial colonization with Pseudomonas aeruginosa postpones chronic infection and prevents deterioration of pulmonary function in cystic fibrosis. Pediatr Pulmonol 1997; 23: 330-335.

27 Taccetti G, Campana S, Festini F, et al. Early eradication therapy against Pseudomonas aeruginosa in cystic fibrosis patients. Eur Respir J 2005; 26: 458-461.

28 Roberts HR, Wells AU, Milne DG, et al. Airflow obstruction in bronchiectasis: correlation between computed tomography features and pulmonary function tests. Thorax 2000; 55: 198-204.

29 Kang EY, Miller RR, Muller NL. Bronchiectasis: comparison of preoperative thin-section CT and pathologic findings in resected specimens. Radiology 1995; 195: 649-654.

30 Thurlbeck WM. Chronic airflow obstruction. In: Thurlbeck WM, Chung AM, eds. Pathology of the Lung. 2nd Edn. New York, Thieme Medical Publishers, 1995; pp. 739-825. 